Zur ökonomischen Spezifizierung des Leitbilds der Nachhaltigen Entwicklung

\section{Ökologisch-ökonomische Perspektive}

\section{Die ökonomischen Nachhaltigkeitsregeln der Enquete-Kommissíon basieren auf einem neoklassischen Marktkonzept und einem wohlfahrtstheoretischen Verständnis politischer Eingriffsmöglichkeiten. Aus einem Blickwinkel der Öko- logischen Ökonomie erscheinen die Regeln problematisch. Abschließend werden Ansätze einer alternativen Formulierung skizziert.}

A

Von Frank Beckenbach us dem Abschlußbericht der EnqueteKommission (EK) lassen sich zwei verdienstvolle Erweiterungen der bisherigen Nachhaltigkeitsdebatte entnehmen: Erstens geht es um die Erweiterung einer allein oder überwiegend ökologiebezogen geführten Nachaltigkeitsdiskussion um ausdifferenzierte ökonomische und soziale Kriterien. Implizit in ökologische Nachhaltigkeitskonzepte eingehende Wertvorstellungen und Normenpostulate werden so transparent gemacht.

Zweitens soll diese Erweiterung offenbar unter Berücksichtigung der für ökonomische und soziale Prozesse eigentümlichen Dynamik erfolgen. Damit geht die Anerkennung einer Offenheit bzw. dauerhaften Unabgeschlossenheit des ökonomischen Prozesses einher (sichtbar etwa an den immer wieder stattfindenden Innovationsvorgängen). Die unterschiedlichen Zeitskalen, in denen sich ökologische, ökonomische und soziale Veränderungen vollziehen, können so Berücksichtigung finden.

Unklar bleibt jedoch, ob das Bemühen der Enquete-Kommission ganz generell auf Aussagen über die Zukunftsbeständigkeit der ökonomischen Verkehrsformen (bzw. ihrer institutionellen Ausgestaltung) und der sozialen Regelungsmechanismen zielt oder aber - mit wesentlich eingeschränkterer Perspektive auf die 'ökonomieverträglichkeit' der bisher in der Nachhaltigkeitsdiskussion behandelten ökologie- und gerechtigkeitsbezogenen Ziele. Sollte die erstgenannte Option gegeben sein, läuft dies auf eine hoffnungslose Überfrachtung der Nachhaltigkeitsdiskussion hinaus. Ich werde daher im folgenden lediglich die letztgenannte Option als Fragestellung voraussetzen.
Die v.a. von Herman Daly formulierten und von der Enquete-Kommission weiterentwickelten ökologischen Nachhaltigkeitsregeln (1) stellen (mehr oder weniger) operationalisierte Verhaltensanweisungen dar, die unter definierten Bedingungen befolgt werden sollen. Diese Regeln sind aus präskriptiven Hintergrundsüberlegungen abgeleitet. Im Falle der Nachhaltigkeitsregeln Dalys sind diese Hintergrundshypothesen die Endlichkeit der Ressourcen, die Komplementarität zwischen natürlichen und menschengemachten Ressourcen und ein Pessimismus in Bezug auf die Möglichkeiten des technischen Wandels. Im Unterschied dazu sind die von der EnqueteKommission vorgeschlagenen ökonomischen Nachhaltigkeitsregeln (mit Ausnahme der Regel 4) diese präskriptiven Hintergrundsüberlegungen selbst und eigentlich keine Regeln im obigen Sinn

\section{Ökonomische Scheingewißheiten}

Den Kern dieser präskriptiven Hintergrundsüberlegungen bildet ein neoklassisches Marktkonzept und ein wohlfahrtstheoretischer Zuschnitt der Aufgabenstellung staatlicher Aktivitäten. In dieser Sichtweise ist die Ökonomie eine Veranstaltung zur effizienten Allokation knapper Ressourcen, in der den Preisen (für Güter, Produktionsfaktoren etc.) eine zentrale Signal- und Koordinationsfunktion zukommt. Staatliche Aktivitäten sind als Korrektiv dann erforderlich, wenn die effiziente Ressourcenallokation behindert wird, z.B. bei wettbewerbshindernden Konzentrationsprozessen und externen Effekten. Die damit einhergehende Option einer weitgehenden ressourcenpolitischen Abstinenz des Staates beruht auf dem Postulat, daß, die Preise die wesentliche Lenkungsfunktion auf Märkten wahrnehmen“ (EK,
S. 26) und entsprechend auch die Knappheiten der ökologischen Quellen- und Senkenressourcen anzeigen sollen.

Gegen diese Vision sollen hier zwei Einwände formuliert werden: Erstens unterliegt ihr (in Regel 1 und 2) eine naive Vorstellung über die Bildung von Preisen in einer modernen Volkswirtschaft. Soweit die ökologischen Ressourcen überhaupt einer Preisbildung zugänglich sind und Preise ein Indikator für Ressourcenverfügbarkeiten sind, etwa auf den Rohstoffmärkten, zeigen sie diese Verfïgbarkeiten für einen relativ kurzfristigen (über weniger als zehn Jahre reichenden) Horizont an. Sie liegen damit weit unter dem Zeitraum, der für einen ressourcenersetzenden technischen Wandel erforderlich ist. Preise sind aber darüberhinaus auch ein Indikator für diverse andere Sachverhalte, die unmittelbar nichts mit der (Ressourcen-)Knappheit zu tun haben: die „Liquiditätspräferenz“ (Keynes), den geld- und kreditpolitischen Kontext für die jeweiligen Anbieter und Nachfrager und last but not least für Input-/ Outputverflechtungen.

\section{Inkonsistente Vision}

Zweitens wird die Markt- und Wohlfahrtsvision inkonsistent, wenn - wie mit der „Regel“ 3 (EK, S. 26) - der innovative Suchprozeß der Marktakteure zu einer ökonomischen Nachhaltigkeitsbedingung werden soll. In einer Innovationsökonomie ist der Informationsstand über künftige Zustände notwendigerweise unvollständig. Die ökonomische Forschung hat sowohl theoretisch plausibilisiert als auch empirisch belegt, daß unter diesen Bedingungen positive Rückkopplungen in Gestalt von learning by using, Netzwerkexternalitäten und steigenden Skalenerträgen der Produktion auftreten (2). Dann ist es möglich, daß sich - bedingt durch zufällige Ausgangskonstellationen oder exogene Störungen - Produktions- und Handlungsalternativen festsetzen, die nicht optimal sind. Pfadabhängigkeit kann so zum „lock-in“ führen; ein Beispiel ist Windows 95 bzw. 98. Dazu kommt, daß mit der Einbettung der ökonomischen Aktivitäten in die ,umgebenden Ökosysteme" (EK, S. 21) aufgrund deren allfälliger Komplexität (wie etwa im Falle von anthropogenen Klimaveränderungen und ihren sozioökonomischen Folgen) die Existenz von negativen Externalitäten zu einem Dauerphänomen wird. Mit deren „effizienter“ Internalisierung wäre jede staatliche oder suprastaatliche Agentur hoffnungslos überfordert. 
Nimmt man diese beiden Gesichtspunkte (ökonomische Innovation und ökologische Komplexität) zusammen, dann ist es keineswegs ausgemacht, daß die Lernfähigkeit sich selbst überlassener Marktakteure hinreicht, um die (ökologisch erforderliche) Nachhaltigkeit sicherzustellen. Kurz gesagt: mit der Existenz von ökonomischen und ökologischen Komplexitäten wird die effiziente Allokation der Ressourcen und deren wohlfahrtsstaatliche Flankierung zu einem ziemlich unwahrscheinlichen Sonderfall. Es drängt sich daher die Vermutung auf, daß ein ausschließlich effizienztheoretisches Marktkonzept und ein ausschließlich wohlfahrtstheoretisches Regelungskonzept nicht den Bereich abdecken, der für die Ableitung ökonomischer Nachhaltigkeitsregeln berücksichtigt werden muß.

In Regel 4 schließlich wird die aus der Ökologischen Ökonomie bekannte Forderung nach der ,Erhaltung des Naturkapitals“ auf das ökonomische Kapital im weiteren Sinne übertragen und die Erhaltung des „Produktiv-, Sozialund Humankapitals“ als auch der ,Leistungsfähigkeit der Gesellschaft" gefordert (EK, S. 27). Die sich bereits bei der Forderung der Erhaltung des Naturkapitals stellenden Probleme werden mit dieser Erweiterung potenziert: Erstens ist im Rahmen der auch von der Enquete-Kommission vorgeschlagenen dynamischen Betrachtungsweise nicht einzusehen, warum für die Sicherstellung einer Flußgröße (Leistungsfähigkeit, Güter- und Dienstleistungserstellung) die Erhaltung der Bestandsgöße Kapital erforderlich sein soll.

Dem stehen schon allein die mit der postulierten Innovation notwendigerweise verbundenen Entwertungseffekte (von Produktionsanlagen, Qualifikationen und Institutionen) entgegen. Zweitens ist schleierhaft, wie das Aggregat „Produktiv-, Sozial- und Humankapital" in irgendeiner sinnvollen Weise meßbar gemacht werden soll. Mag die Ermittlung eines 'realen' Bewertungsindikators für das Anlagevermögen noch mit Hilfe einiger statistischer Tricks möglich sein, bei der Ermittlung des Aggregats Humankapital wird man ohne die spekulativen Konstrukte der ökonomischen Theorie nicht auskommen (welches wird genommen?). Die Bestimmung des Sozialkapitals unter Einschluß der personellen und institutionellen Infrastruktur wird ein frommer Wunsch bleiben.

\section{Systemdynamik und Nachhaltigkeitsfenster}

Aus der obigen Kritik läßt sich als erste Folgerung ziehen, daß sich Nachhaltigkeitsregeln sinnvoll nur in einem systemisch-dynamischem Rahmen formulieren lassen. An die Stelle der Fokussierung auf Bestandsgrößen („Kapital“ jedweder Art) tritt dann die Fokussierung auf die Veränderung dieser Zustandsgrößen (bedingt durch Flußgrößen wie die Nettoproduktion von Biomasse oder die Nettoanlageinvestitionen). Dem entspricht zum einen, daß die zwischen den verschiedenen Flußgrößen bestehenden Interdependenzen berücksichtigt werden und zum andern, daß die zeitabhängige Zustandsfolge dieser Flußgrößen (die 'Lösung' des Systems) bedeutsam werden. An die Stelle des Postulats der „Erhaltung“ einer Bestandsgröße tritt dann das Postulat der Vermeidung abrupter Sprünge oder der Aufrechterhaltung eines dauerhaften und robusten Zustandes dieser Flußgrößen.

Die zweite Folgerung ist, daß eine echte Verknüpfung der ökologischen und ökonomischen Nachhaltigkeitsregeln erforderlich ist. Hier soll der Vorschlag gemacht werden, diese Verknüpfung in zwei Schritten anzugehen:

1. durch die Formulierung von ökologischen und ökonomischen Mindestbedingungen für das Überleben moderner, auf Märkte zentrierter Gesellschaften und

2. durch die Übersetzung dieser Mindestbedingungen in ökologisch-ökonomische Nachhaltigkeitsfenster.
Beispiele für ökologische Mindestbedingungen und damit Schranken für die ökonomische Nutzung sind die Sicherstellung von Mindestwerten für die Regulations-, Träger- und Produktionsfunktion von ökologischen Systemen, die Klimaund Klimazonenstabilität (Erhaltung des ocean conveyor belt, keine Veränderung der Monsunbzw. Jetstreamdynamik etc.) und die Aufrechterhaltung des Nährstoffrecyclings (3).

Ökonomische Nachhaltigkeitsessentials könnten demgegenüber sein:

- die Sicherstellung einer 'ausreichenden' Ressourcenverfügbarkeit (Aufrechterhaltung der ökonomischen Tätigkeitsdifferenzierung, keine prohibitiven Preisschwankungen der Ressourcenpreise),

- die Sicherstellung der Innovationsfähigkeit (keine prohibitive Folgekosteninternalisierung), - die Sicherstellung der Legitimität und Effektivität von Institutionen (keine Folgekostendramatisierung, Vermeidung von großen Migrationsbewegungen) und

- die Aufrechterhaltung stabiler ökologischer Nutzungsregime (akzeptierte Zuweisung von Eigentumsrechten).

Unter der Voraussetzung, daß den ökonomischen Aktivitäten sowohl jeweils eingrenzbare Werte für die Ressourcenentnahme als auch für die Ressourcenrückgabe in Gestalt von Emissionen und Abfall zugeordnet werden können (Extraktionsund Insertionsfunktionen) und daß es möglich ist, für die jeweiligen Werte der Extraktions- und Insertionsfunktion die qualitative Lösung des ökologischen Systems zu spezifizieren, lassen sich dann ökologisch-ökonomische Nachhaltigkeitsfenster

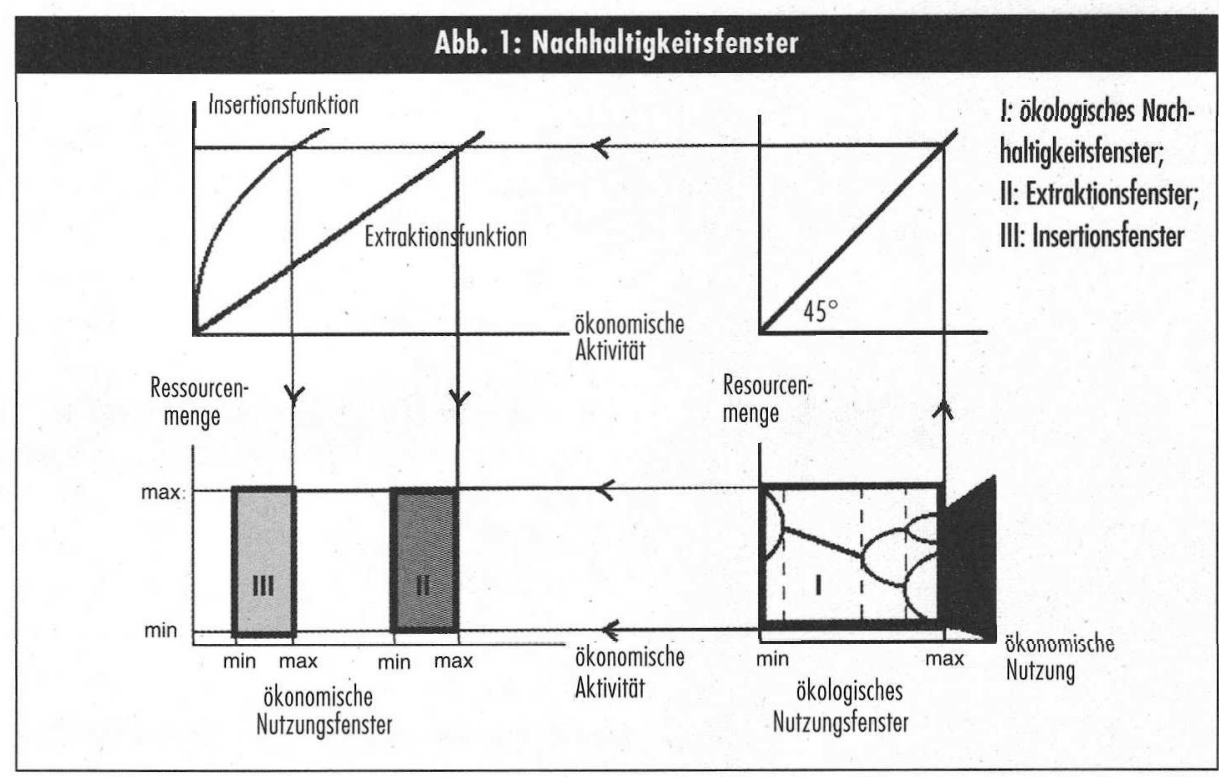

Quelle: Eigene Darstellung 
eingrenzen (vgl. Abb. 1). Dabei entsprechen den ökologischen Mindestbedingungen ökonomische Nutzungsobergrenzen; demgegenüber stellen die ökonomischen Mindestbedingungen die ökonomischen Nutzungsuntergrenzen dar. Innerhalb des ökologischen Nachhaltigkeitsfensters lassen sich unterschiedliche Lösungsregime (Gleichgewicht, Zyklen unterschiedlicher Länge) abgrenzen (..... Linien in I), innerhalb derer das System jeweils resilient, d.h. stabil in Bezug auf die ökonomische Nutzung ist.

Es kann hier abschließend nur darauf verwiesen werden, daß die marktendogenen Innovationsprozesse für eine beständige Veränderung der Extraktions- und Insertionsfunktionen sorgen. Ebenso ist die Verständigung auf ökologische und ökonomische Mindestbedingungen nicht allein durch wissenschaftliche bzw. ethische Überlegungen herzustellen, sondern bedarf der Abklärung mit dem faktischen Prozeß der Artikulation und Verarbeitung ökologischer Knappheit in der Gesellschaft. Gleichwohl stellen wissenschaftliche und ethische Überlegungen ein Element dieses Prozesses dar (4).

\section{Anmerkungen}

(1) Vgl. z.B. Daly, Herman: Towards Some Operational Principles of Sustainable Development. Ecological Economics, Vol. 2 (1990), S. 1-6.

(2) Vgl. z.B. Arthur, W. B.: Positive Rückkopplung in der Wirtschaft. Spektrum der Wissenschaft 1990, S. 122-129.

(3) Vgl: für eine ausführliche Diskussion z.B.

De Groot, R. S.: Functions of Nature. Amsterdam: WoltersNoordhoff 1992, S. 13ff.,

Costanza, R. et al.: An Introduction to Ecological Economics. Boca Raton: St. Lucie Press 1997 und

Schellnhuber, H. J.: Integrated Assessment of Climate Change: Regularity and Singularity. Potsdam: Potsdam Institut für Klimafolgenforschung 1998, S. 8 f.

(4) Vgl. Beckenbach, F.: Beschränkte Rationalität und Systemkomplexitöt. Ein Beitrag zur Ökologischen Ökonomik. Marburg: Metropolis 1998

\section{Der Autor}

Dr. Frank Beckenbach ist Hochschulassistent für Sozioökonomie an der Universität Osnabrück. Kontakt: Universität Osnabrück, FB Sozialwissenschaften, 49069 Osnabrück. Tel. 0541/ 969-4659, Fax-4600, E-mail: fbeckenb@rz.uni-osnabrueck.de

Zum Verhältnis von monetärem Sektor und Nachhaltigkeit

\title{
Konflikt zwischen Geld und Natur?
}

\author{
Die Umweltproblematik wird bislang in aller Regel als ausschließlich reales \\ Phänomen gesehen, bei dem die Institution "Geld" keine Rolle spielt. Es lassen \\ sich jedoch plausible Gründe anführen für einen vom Geld- und Kreditwesen \\ induzierten Wachstumsautomatismus, der langfristig in Konflikt mit dem Ziel \\ einer Nachhaltigen Entwicklung, d.h. einer dauerhaften Erhaltung der natürli- \\ chen Lebensgrundlagen, steht. Dies hat Konsequenzen für die Formulierung der \\ ökonomischen Dimension der Nachhaltigkeit.
}

B ei der mischer Fragestellungen ist es durchas zulässig und sinnvoll, von der Neutralität des Geldes auszugehen. Es ist jedoch fraglich, ob dies auch bei einer grundsätzlichen Betrachtung des Verhältnisses zwischen Ökonomie und Ökologie gemacht werden kann - wie dies offensichtlich auch die Enquete-Kommission „Schutz des Menschen und der Umwelt“ tut, in deren ökonomischem Regelwerk monetäre Faktoren gänzlich außer acht gelassen werden (1). Mit den zwischen Geldsystem und Umweltproblem bestehenden Zusammenhängen hat sich vor allem H.C. Binswanger beschäftigt (2). Der Ausgangspunkt seiner Überlegungen ist die von Aristoteles (Politik, 1256a-1257b) behauptete Grenzenlosigkeit der Gelderwerbskunst.

\section{Dynamik des Geldes}

Diese im Wesen des Geldes liegende Maßlosigkeit konnte jedoch erst in modernen Geldverfassungen zur Entfaltung kommen. Erst durch die Einführung stoffwertlosen und schließlich stofflosen Geldes entielen die früher bestehenden angebotsseitigen Grenzen der Geldexpansion und die Geldmenge konnte tatsächlich unbegrenzt vermehrt werden. Aber H.C. Binswanger geht wesentlich weiter: Nach seiner Meinung ermöglicht das moderne Geldwesen die Geldexpansion nicht nur, es erzwingt sie sogar. Dieser Expansionszwang wird zurïckgeführt auf die Kapitalisierung des Geldes, d.h. die Verwendung des Geldes auch zu Investitionszwecken und die Schaffung eines Großteils des Geldes durch Vergabe von Krediten, insbesondere von Investitionskrediten. Er manifestiert sich vor allem auf der makroskopischen Ebene und zwar auf zweierlei Art und Weise. Einerseits behält das Geld, insoweit es durch die Kreditver- gabe der Geschäftsbanken an Unternehmen entsteht, seinen Wert bzw. seine Geltung nur dann, wenn die durch diese Kredite finanzierten Investitionen erfolgreich sind, also die Unternehmen - zumindest per Saldo - Gewinne erzielen; dies ist wiederum nur dann möglich, wenn Geld neu zufließt, also die Kreditvergabe zunimmt. Der Bestand des ,alten“ Geldes hängt also von der ständigen Schöpfung ,neuen“ Geldes ab! Andererseits tritt bei Investitionen zuerst der Einkommens- und dann der Kapazitätseffekt auf, so daß die Mehrproduktion aufgrund der in der Vorperiode getätigten Investitionen nur dann abgesetzt werden kann, wenn ihr in der aktuellen Periode eine entsprechende Einkommenssteigerung gegenübersteht, also erneut Investitionen getätigt werden.

Diese immanente Dynamik unseres Geldwesens erfährt eine mikroökonomische Ergänzung durch die Kapitalisierung erwarteter Gewinnsteigerungen. Der Gegenwartswert von Unternehmensanteilen wird hauptsächlich durch die erwarteten künftigen Gewinne der betreffenden Unternehmen determiniert. Werden nicht nur konstante, sondern auf Dauer steigende Gewinne unterstellt, so ergibt sich eine beträchtliche Erhöhung des Wertes der Anteile. Dieser Wert kann jedoch nur bei einer tatsächlichen Realisierung des antizipierten permanenten Gewinnzuwachses Bestand haben. Kommt es nur zu einer Stagnation der Gewinne (und nicht etwa schon zu Verlusten!), so erleiden die Anteilseigner einen Wertverlust. Da die Anteilseigner offensichtlich einen solchen vermeiden wollen, liegt es in ihrem Interesse, daß ständig der Gewinn erhöht wird, also ständig Nettoinvestititonen getätigt werden und so der Wachstumsprozeß in Gang gehalten wird. „Darum ist die Wirtschaft heute zum Wachstum 'verurteilt' nicht weil die Menschen unersättlich sind, son- 
(c) 20I0 Authors; licensee IÖW and oekom verlag. This is an article distributed under the terms of the Creative Commons Attribution Non-Commercial No Derivates License (http://creativecommons.org/licenses/by-nc-nd/3.o/), which permits unrestricted use, distribution, and reproduction in any medium, provided the original work is properly cited. 